DISKUSSIONSFORUM

\section{Technology Assessment und Wirtschaft: Transparenz tut Not!}

\author{
von Utz Schäffer, WHU Koblenz - Otto \\ Beisheim Hochschule
}

Im Heft 2/2001 der TA-Datenbank-Nachrichten wurde das Schwerpunktthema „Technikfolgenabschätzung und Industrie“ kontrovers diskutiert. Da in diesem Zusammenhang mehrfach auf die WHU-Studie (Weber et al. 1999) verwiesen wurde (vgl. Baron und Zweck 2001, S. 9; Astor und Giesecke 2001, S. 16; Fürstenwerth 2001, S. 25), möchten auch wir uns an dieser Stelle zu Wort melden und den Diskussionsfaden aufgreifen. Dies soll im Folgenden in Form von vier Hypothesen geschehen. Wir laden die Community dazu ein, kritisch oder zustimmend Stellung zu nehmen.

\section{Hypothese 1: Es weht ein frischer Wind in der TA-Landschaft, nachhaltige Verände- rungen sind aber noch nicht erreicht.}

Vor geraumer Zeit nahm die WHU-Studie eine Bestandsaufnahme und Analyse technikreflektierender Forschung in Deutschland sowie eine Ableitung von Gestaltungs- und Handlungsempfehlungen vor. Eine der zentralen Konklusionen lautete:

\section{„Wir empfehlen daher eine konsequente Rückführung der institutionellen Förderung und das ,Aufbrechen' der weitgehend in sich geschlossenen Community durch eine Reihe von konkreten Maßnahmen zur Förderung von Transparenz und Wettbewerb, insbeson- dere auch der stärkeren Integration von Un- ternehmen in den Prozess." (Weber et al. 1999, S. 239)}

Es hat uns in der Folge sehr gefreut, dass eine gewisse Bewegung in der Community zu verzeichnen ist. Neben zahlreichen Diskussionsbeiträgen (insbesondere im Diskussionsforum der TA-Datenbank-Nachrichten) ist zu konstatieren, dass das Bundesministerium für Bildung und Forschung mit der Innovations- und Tech- nikanalyse (ITA) eine Neuakzentuierung seiner Förderaktivitäten vollzogen hat, die unter anderem deutlich machen soll, dass die Analyse von möglichen Technikfolgen nicht losgelöst von Unternehmen erfolgen kann (vgl. Brüntink 2001; Astor und Giesecke 2001).

Zur aktuellen Diskussion seien zwei Anmerkungen erlaubt: Zum einen erscheint uns die intensive Debatte um Sinn oder Unsinn des ITA-Begriffs nicht in hohem Maße nutzbringend $\mathrm{zu}$ sein. Sie bringt per se keine neuen Lösungen und kann in Bezug auf das Außenbild technikreflektierender Forschung eher kontraproduktiv sein. Die Begrifflichkeit ist unseres Erachtens eine Frage des Marketing und damit sicher nicht zu vernachlässigen. Sie darf aber nicht den Blick auf die inhaltlichen Fragen verstellen (vgl. dazu auch nochmals Weber et al. 1999). Zum anderen wird unseres Erachtens eine Bereitschaft, sich mit den Positionen der jeweils anderen Diskussionsteilnehmer inhaltlich auseinander zu setzen, leider nicht in allen Beiträgen der aktuellen Diskussion sichtbar. Zudem erscheint die Debatte (bei aller notwendigen Pointierung der Argumentation) nicht immer ausreichend sachlich orientiert zu sein. Vor diesem Hintergrund stimmen wir der Analyse von Grunwald zu:

\footnotetext{
„Abgehobene Debatten der Art um Sinn oder Unsinn von TA, ob TA als Politikberatung oder in der Industrie betrieben werden sollte, begeben sich aber rasch auf polemisches Glatteis und werden irrevelevant: Austausch von allgemeinen Weltanschauungen statt belastbare Argumentation." (Grunwald 2001; vgl. auch die Diskussion Grunwald 2000 und Weber und Schäffer 2000)
}

Die vom BMBF initiierten Workshops und die Berliner Konferenz ,Innovations for an E-Society. Challenges for Technology Assessment" sowie der deutsche Forschungsdialog (Futur) [siehe hierzu die Beiträge in diesem Heft] $\mathbf{e}$ hen wir als wichtige Maßnahmen zur Unterstützung einer entsprechenden Öffnung der institutionalisierten TA in Deutschland. Allein: die Notwendigkeit zu Veränderungen ist nicht überall erkannt (oder zumindest nicht in die Tat umgesetzt). Dazu bedarf es neben einer intensiven und fundierten Diskussion über Kosten und Nutzen verschiedener TA-Dienstleistungen der stärkeren Integration geeigneter Markt- und Wettbewerbsmechanismen in die Community. 
Die Aussage der WHU-Studie, dass „weite Teile der TA-Anbieterschaft nicht dem Konkurrenzdruck ausgesetzt sind und auf stetige Alimentierung durch die öffentliche Hand setzen können“" (Weber et al. 1999, S. 185f.), gilt im Kern noch immer. Nachhaltige Veränderungen setzen geeignete Anreize voraus. Durch Diskussionsprozesse allein wurden verfestigte Marktstrukturen noch selten aufgelöst.

\section{Hypothese 2: Die aktuelle Diskussion über TA in, mit und für die Wirtschaft stößt an Grenzen. Es bedarf einer empirischen Basis.}

Die folgenden Behauptungen bezüglich TA und Wirtschaft scheinen uns mittlerweile unstrittig zu sein: (1) Technologieentwicklung wird im Wesentlichen in der Wirtschaft betrieben (vgl. z.B. Astor und Giesecke 2001, S. 20); (2) die Wirtschaft sollte stärker in die traditionelle TA (als Politikberatung) einbezogen werden (vgl. Brüntink 2001, S. 8; Baron und Zweck 2001; Astor und Giesecke 2001; Fürstenwerth 2001) und (3) auch in der Wirtschaft scheint es „TA-ähnliche Beratungsprozesse" (Grunwald 2001, S. 69) zu geben (vgl. auch die Beiträge von Acker-Widmaier und Maring 2001 und Minx 2001). Die empirische Basis bezüglich Technology Assessment in der deutschen Wirtschaft ist aber vergleichsweise dünn (vgl. Fleischmann und Paul 1987; Skrotzki 1989 und Diery 1996). Vor diesem Hintergrund überrascht es nicht, dass in der aktuellen Diskussion konträre Einschätzungen über das Vorhandensein von TA in der Wirtschaft nebeneinander stehen (vgl. Grunwald 2001, S. 69 und Baron und Zweck 2001, S. 9 und ebenda, S. 15). Insbesondere bezüglich kleiner und mittelständischer Unternehmen sowie Start-Ups liegt bislang wenig verlässliches Wissen vor.

Im nächsten Schritt soll daher eine Bestands- und Bedarfsaufnahme der TA mit, in und für die Wirtschaft vorgenommen werden (vgl. auch ähnlich Grunwald 2001, S. 64). Zudem gilt es, soweit wie möglich Transparenz über Kosten und Nutzen derartiger Aktivitäten zu gewinnen. Eine deskriptive Aufnahme der TA-Aktivitäten im Status Quo ist zwar hilfreich und wichtig, greift aber letztlich zu kurz. Eine aktuelle Studie der WHU (in Kooperation mit dem VDI-Technologiezentrum Düsseldorf) setzt an dieser Stelle an und versucht, durch eine Reihe von Fallstudien und Interviews einen ersten Überblick über TA in der Wirtschaft zu geben. Sie will so die Diskussion in der Community (und darüber hinaus!) befruchten und die notwendige Basis für eine darauf aufbauende großzahlige schriftliche Erhebung bilden.

Und noch ein letzter Punkt: Auch ein Blick über die Grenzen zeigt interessante Ansätze bezüglich TA in, mit und für die Wirtschaft, die auch in Deutschland Sinn machen könnten. Beispielhaft sei nur auf das „NTTC“ (National Technology Transfer Center) in den USA verwiesen (vgl. auch Häußler et al. 2001).

\section{Hypothese 3: Beschäftigt man sich mit TA und Wirtschaft, erscheint eine differenzierte Betrachtung essenziell. Das Schlagwort (I)TA ist zu vielschichtig und unscharf.}

Bei der empirischen Analyse von TA und Wirtschaft gilt es - soweit möglich - differenziert vorzugehen:

- Im ersten Schritt kann unterschieden werden, ob TA in, mit oder für die Wirtschaft erfolgt. Dies entspricht der betriebswirtschaftlichen Unterscheidung ,make“, „cooperate“ oder „buy“ als alternative Strategien der Unternehmen.

- Daneben erscheint eine Unterscheidung verschiedener Unternehmenstypen (Großunternehmen, KMUs, Start-Ups) sowie unterschiedlicher Branchen sinnvoll. Insbesondere die (möglicherweise) spezifischen Bedürfnisse von KMUs und StartUps (vgl. Weber et al. 1999 und Brüntink 2001, S. 8) wurden bislang nicht näher betrachtet (vgl. zu TA und Start-Ups auch Häußler et al. 2001).

- Schließlich kann in Anlehnung an die WHU-Studie nach den Funktionen, die der TA im Unternehmen zukommen, unterschieden werden. Im Rahmen der Willensbildung sind insbesondere die technologische Früherkennung (vgl. etwa Jischa 2001, S. 24) und die Produktfolgenabschätzung zu nennen (vgl. Schade 1988 sowie Minx und Meyer 2001, S. 41 und ebenda, S. 44.). Daneben kann TA auch im Rahmen der Willensdurchsetzung bzw. Kommunikation, Lobbyismus und Ak- 
zeptanzgewinnung von Nutzen sein (vgl. etwa Becks und Gelbke 2001, S. 34f.)

In der aktuellen Diskussion beobachten wir vielfach Kommunikationsdefizite, weil $\mathrm{Ge}$ sprächspartner ein unterschiedliches Tätigkeitsfeld im Auge haben. Man vergleiche nur die Aktivitäten des Forschungszentrums von Professor Minx bei der DaimlerChrysler AG und die TA-relevanten Aktivitäten eines betrieblichen Umweltschutzbeauftragten. Zudem besteht kein Konsens über die relative Bedeutung der einzelnen Funktionen von TA in Unternehmen (vgl. auch Grunwald 2001, S. 63). Angesichts dieser Sachverhalte erscheint eine undifferenzierte Diskussion über TA und Unternehmen wenig hilfreich.

\section{Hypothese 4: Unabängige Politikberatung und die verstärkte Interaktion von TA- Institutionen mit Unternehmen schließen sich nicht aus - im Gegenteil: positive Rückkopplungen sind möglich.}

Folgt man Teilen der aktuellen Diskussion, steht die Community am Scheideweg zwischen einer am Gemeinwohl orientierten und unabhängigen TA auf der einen Seite und einer Partikularinteressen verfallenen Beratungstätigkeit in enger Interaktion mit der Wirtschaft auf der anderen Seite (vgl. Grunwald 2000, S. 132f.; Weber und Schäfer 2000, S. 155f.; Astor und Giesecke 2001, S. 16 und Fürstenwerth 2001, S. 28f.). Diese dichotome Sicht führt jedoch in die Irre: Zum einen schafft gerade die Ausrichtung auf einen oder wenige staatliche oder halbstaatliche Adressaten Abhängigkeiten: ein vielschichtiges, differenziertes Kundenspektrum sollte im Gegenteil die auch heute massiv bestehenden und beobachtbaren $\mathrm{Ab}$ hängigkeiten reduzieren helfen (vgl. dazu nochmals Weber et al. 1999 sowie Astor und Giesecke 2001, S. 18f). Zum anderen beziehen führende Unternehmensberater in der Wirtschaft ihre Reputation und damit die notwendige Voraussetzung für ihren Markterfolg aus der Unabhängigkeit und ihrer Bereitschaft, auch unangenehme Wahrheiten zu kommunizieren (sowie auf die Umsetzung ihrer Erkenntnisse hinzuwirken).

An die Stelle eines verkürzten ,entweder ... oder" sollten vielmehr ,gegenseitiges Lernen“ (Grunwald 2001, S. 63) und ein ,sowohl
... als auch" treten: Wenn TA wieder im Zentrum der gesellschaftlichen Zukunftsdiskussion stehen will (vgl. Grunwald 2000, S. 133; Weber und Schäffer 2000, S. 155 und Fürstenwerth 2001, S. 25), muss sie sich die „Finger schmutzig machen" und (auch) in enger Interaktion mit Unternehmen stehen, die einen ganz wesentlichen Beitrag dazu leisten, Technologien $\mathrm{zu}$ entwickeln und voranzutreiben (vgl. auch Astor und Giesecke 2001, S. 20). Aus dem dort generierten Wissen können wiederum positive Rückkopplungen in die Politikberatung entstehen.

\section{Literatur}

Acker-Widmaier, G.; Maring, M., 2001: Kompetenzzentrum/-netzwerk „Technikbewertung im Unternehmen“. TA-Datenbank-Nachrichten, 10. Jg., Nr. 2/2001, S. 50-51.

Astor, M.; Giesecke, S., 2001: Innovationen für die TA. TA-Datenbank-Nachrichten, 10 . Jg., Nr. 2/2001, S. 16-20.

Baron, W.; Zweck, A., 2001: Bedarf und Nutzen von ITA für die Wirtschaft. TA-Datenbank-Nachrichten, 10. Jg., Nr. 2/2001, S. 9-15.

Becks, H.; Gelbke, H.-P., 2001: Die ÖkoeffizienzAnalyse nach BASF. TA-Datenbank-Nachrichten, 10. Jg., Nr. 2/2001, S. 34-38.

Brüntink, C., 2001: Zum Konzept der Innovationsund Technikanalyse des BMBF. TA-DatenbankNachrichten, 10. Jg., Nr. 2/2001, S. 6-8.

Diery, H., 1996: Technologiefolgen-Abschätzung als strategische Aufgabe einer prospektiven Arbeitsund Organisationsgestaltung. Frankfurt a. M.

Fleischmann, G.; Paul, I., 1987: TechnikfolgenAbschätzung in der Industrie der Bundesrepublik Deutschland. Frankfurt a.M.

Fürstenwerth, H., 2001: Think different - just do it! TA-Datenbank-Nachrichten, 10. Jg., Nr. 2/2001, S. 25-33.

Grunwald, A., 2000: TA - Politikberatung oder Unternehmensberatung? Anmerkungen $\mathrm{zu}$ einer aktuellen Diskussion. TA-Datenbank-Nachrichten, 9. Jg., Nr. 3/2000, S. 132-138.

Grunwald, A., 2001: Arbeitsteilige Technikgestaltung und verteilte Beratung: TA zwischen Politikberatung und Technikbewertung im Unternehmen. TA-Datenbank-Nachrichten, 10. Jg., Nr. 2/2001, S. 61-71.

Häußler, S.; Schäffer, U.; Weber, J., 2001: Innovation and Technology Analysis (ITA) in German Start-Ups. In: Institut für Technikfolgenabschätzung und Systemanalyse und VDI/VDE-Technologiezentrum Informationstechnik (Hrsg.): Innovations for an e-Society. Challenges for Technology Assess- 
ment. Congress Pre-prints, Teltow 2001, Part I, Session 7, ohne durchnummerierte Seitenzahlen.

Jischa, M.F., 2001: Managementsysteme für die Wirtschaft. TA-Datenbank-Nachrichten, 10. Jg., Nr. 2/2001, S. 21-24.

Minx, E.; Meyer, H., 2001: Produktfolgenabschätzung im Rahmen des Innovationsmanagements. TA-Datenbank-Nachrichten, 10. Jg., Nr. 2/2001, S. 39-44.

Schade, D., 1988: Technikfolgenabschätzung im Staat, Produktfolgenabschätzung in der Wirtschaft. In: Daimler-Benz AG (Hrsg.): Technikfolgenabschätzung und Technikbewertung. Konzeption, Anwendungsfälle, Perspektiven. Report 10, Düsseldorf, S. 7-14

Skrotzki, R., 1989: Arbeitswissenschaftliche Technologiefolgen-Abschätzung und Technik-Beeinflussung, Düsseldorf

Weber, J.; Schäffer, U.; Hoffmann, D.; Kehrmann, T., 1999: Technology Assessment - Eine Managementperspektive. Bestandsaufnahme - Analyse Handlungsempfehlungen. Wiesbaden: Gabler 1999. Weber; J.; Schäffer, U., 2000: Können betriebswirtschaftliche Erkenntnisse die TA in Deutschland unterstützen? - Anmerkungen zum Beitrag von Armin Grunwald. TA-Datenbank-Nachrichten, 10. Jg., Nr. 2/2001, S. 154-156.

\section{Kontakt}

Privatdozent Dr. Utz Schäffer WHU Koblenz - Otto Beisheim Graduate School of Management

Burgplatz 2, 56179 Vallendar

Tel.: +49 (0) $261 / 6509-450$

Fax: +49 (0) 261 / 6509 - 479

E-Mail: uschaeff@whu.edu 